\title{
A retrospective study on IVF/ICSI outcome in patients with anti-nuclear antibodies: the effects of prednisone plus low-dose aspirin adjuvant treatment
}

\author{
Qing Zhu', Li Wu', Bo Xu, Mei-Hong Hu, Xian-Hong Tong, Jing-Juan Ji and Yu-Sheng Liu*
}

\begin{abstract}
Background: Anti-nuclear antibodies (ANA) are suspected of having relevance to adverse reproductive events. Methods: This study aims to investigate the potential effect of ANA on IVF/ICSI outcome and the therapeutic role of prednisone plus low-dose aspirin $(P+A)$ adjuvant treatment in ANA + patients. The first IVF/ICSI cycles without $\mathrm{P}+\mathrm{A}$ of sixty-six ANA + women were enrolled as the ANA + group, and the 233 first IVF/ICSI cycles of matched ANA- women served as the ANA- group. The ANA + group was divided into the Titre $<=1: 320$ subgroup and the Titre $>$ 1:320 subgroup. Twenty-one ANA + women with adverse outcomes in their first cycles (ANA + cycles without $P+A$ ) received $P+A$ adjuvant treatment for three months before the second IVF/ICSI cycle (ANA + cycles with $P+A$ ). The clinical characteristics and the IVF/ICSI outcomes were compared, respectively, between 1) the ANA + group and the ANA- group, 2) the Titre $<=1: 320$ subgroup and the Titre $>$ 1:320 subgroup, and 3 ) the ANA + cycles without $P+A$ and the ANA + cycles with $P+A$.

Results: No significant differences were observed between each of the two-group pairs in the clinical characteristics. The ANA + group exhibited significantly lower MII oocytes rate, normal fertilisation, pregnancy and implantation rates, as well as remarkably higher abnormal fertilisation and early miscarriage rates. The Titre $<=1: 320$ subgroup's IVF/ICSI outcomes were as poor as those of the Titre > 1:320 subgroup. After the P + A adjuvant treatment, the number of two pro-nuclei, perfect embryos and available embryos, and the implantation rate increased significantly.

Conclusions: These observations suggest that ANA could exert a detrimental effect on IVF/ICSI outcome that might not be titre-dependent, and P + A adjuvant treatment could be useful for ANA + patients. This hypothesis should be verified in further prospective randomised studies.
\end{abstract}

Keywords: Anti-nuclear antibody, ANA, IVF/ICSI outcome, Titre, Prednisone, Aspirin

\section{Background}

A portion of infertile patients consistently fail to conceive despite recurrent transfers of high-quality embryos. The mechanism leading to this reproductive failure is still unclear. To investigate this failure, several researchers have focused on the association between autoimmune factors and

\footnotetext{
* Correspondence: shengzhizhongxin@126.com

${ }^{\dagger}$ Equal contributors

Centre for Reproductive Medicine, Department of Obstetrics and Gynecology, Anhui Provincial Hospital affiliated with Anhui Medical University, Hefei, Anhui 230000, China
}

(c) 2013 Zhu et al.; licensee BioMed Central Ltd. This is an open access article distributed under the terms of the Creative Commons Attribution License (http://creativecommons.org/licenses/by/2.0), which permits unrestricted use, distribution, and reproduction in any medium, provided the original work is properly cited.

IVF/ICSI outcome, and the focus is especially directed toward autoantibodies [1-5]. It has been reported that ANA were relevant to adverse reproductive events, including recurrent spontaneous abortion, endometriosis, infertility, IVF failure, and ovarian dysfunction [6-10].

In a broad sense, ANA are a group of autoantibodies that target the entire cell including DNA, RNA and proteins [11]. Most of the common nuclear antigens can be found on the surface structures of apoptotic cells, and these molecules with the phospholipids on the surface of the apoptotic cells can induce an autoimmune reaction, 
resulting in an abnormal release of anti-phospholipids antibodies (APA) or ANA [12,13]. A basic cellular abnormality, such as increased apoptosis or decreased clearance of apoptotic cells might explain the increased APA and ANA levels in individuals with autoimmune diseases [14]. One iconoclastic theory that ANA such as an anti-double strand DNA (anti-dsDNA) antibody, anti-RNP antibody, or anti-ribosomal $\mathrm{P}$ protein antibody could penetrate into living cells has been confirmed in vivo and in vitro [15-17].

It has been suggested that ANA could impair oocyte quality and embryo development, leading to reduced pregnancy and implantation rates $[7,10]$, and women with recurrent pregnancy loss had a significantly higher incidence of ANA than controls [9]. Shirota et al. [18] found that the presence of the anti-centromere antibody might interfere with the oocyte maturation from MI to MII and impair the embryo cleavage potential. Embryo development was severely impaired or failed when cocultured with IgG from ANA + women [19].

In our study, we retrospectively analysed IVF/ICSI outcomes in ANA + women and compared them with ANA-controls. Differing from previous reports, we studied the effects of prednisone plus low-dose aspirin ( $\mathrm{P}+$ A) adjuvant therapy in ANA + women before IVF/ICSI cycles. The aim of this study is to investigate the potential effect of ANA and different ANA titres on IVF/ICSI outcome, and to determine the role of adjuvant treatment in these ANA + patients.

\section{Methods}

\section{Patients}

Patients who received IVF/ICSI treatment were recruited from the Reproductive Medicine Centre of Anhui Provincial Hospital affiliated with Anhui Medical University from October 2009 to September 2012.

In our centre, ANA, D-dimmer, platelet aggregation test, anti-cardiolipin antibody (ACA), anti-beta(2)-glycoprotein I antibody (A- $\beta 2-\mathrm{GPI})$, triiodothyrorine (T3), thyroxine (T4), thyroid stimulating hormone (TSH) and anti-thyroid antibody (ATA) detection examinations were performed to exclude pre-thrombotic state and autoimmune disease. The women positive for ANA and without specific autoimmune diseases were provided with information regarding the $\mathrm{P}+\mathrm{A}$ adjuvant therapy protocol, and after considering the advantages and disadvantages, they decided whether to receive the adjuvant therapy before each cycle.

A total of seventy-three patients meeting the following requirements: 1) with age not over 38 years, 2) with infertility caused by tubal disorder and/or male factor or unexplained infertility, 3) who have undergone IVF/ICSI treatment with the standard long protocol, and 4) positive for ANA and negative for other antibodies were enrolled in our study, wherein seven patients received adjuvant therapy, and the remaining sixty-six patients received no special medications before IVF/ICSI treatment. The first IVF/ICSI cycles (28 IVF cycles and 38 ICSI cycles) of these sixty-six patients were enrolled as the ANA + group.

For the controls, two hundred thirty-three patients 1) with age not over 38 years, 2) with infertility caused by tubal disorder and/or male factor, 3) who have undergone IVF/ICSI treatment with the standard long protocol in the same period as the ANA + patients, 4) negative for any antibody, and 5) who haven't received any special medications before the IVF/ICSI treatment were recruited in our study, and only the first IVF/ICSI cycles (106 IVF cycles and 127 ICSI cycles) were enrolled as the ANA- group.

The exclusion criteria for both groups were as follows: uterine malformation; hypothyroidism; hyperthyroidism; chromosome abnormality of the couples; sexually transmitted diseases (STD) with ureaplasma urealyticum, mycoplasma, chlamydia, gonococcus, fungi, trichomonas vaginalis, HIV or treponema pallidum; or autoimmune diseases such as systemic lupus erythematosus, antiphospholipids syndrome, autoimmune thyroiditis, and Sjogren syndrome. This study was approved by the Ethics Committee of Anhui Provincial Hospital.

According to the ANA titres, the ANA + group was divided into two subgroups, including the Titre $\leq 1: 320$ subgroup (46 cycles) and the Titre $>1: 320$ subgroup (20 cycles). The clinical characteristics and the IVF/ICSI outcomes were compared, respectively, between the ANA + group and the ANA- group, and between the two subgroups.

\section{Adjuvant medications}

Twenty-one of these 66 ANA + patients, who had poor IVF/ICSI outcomes in their first treatment cycles, received a daily oral dose of $10 \mathrm{mg}$ of prednisone plus $100 \mathrm{mg}$ of aspirin up to three months before the second IVF/ICSI cycle, wherein 2 patients (9.5\%) became ANAnegative, and 19 patients (90.5\%) remained ANApositive after treatment. These patients entered the IVF/ ICSI program immediately after three months of adjuvant treatment and received the same long-protocol for controlled ovarian stimulation $(\mathrm{COH})$ as in the first cycles. The one-paired comparison was performed between the first cycles (the ANA + cycles without $\mathrm{P}+\mathrm{A}$ ) and the second cycles (the ANA + cycles with $\mathrm{P}+\mathrm{A}$ ).

\section{ANA assay}

The ANA were detected using the indirect immunofluorescence (IFT) method on the human epithelial (HEp-2) cell substrate. Serum samples from the patients were prepared at various dilution factors as follows: 
$1: 100,1: 320,1: 1000,1: 3200,1: 10000,1: 32000$ and incubated with fixed HEp-2 cells. The ANA could react with the antigens of the Hep-2 cell substrate, forming antigen-antibody complexes bound to the cell nucleus. Temporarily, fluorescein-labelled antihuman immunoglobulin was added and fluorescein-labelled antibodyantigen-antibody complexes were formed and detected by using fluorescence microscopy. The ANA were defined as positive when the signal could be detected in the nucleus with the serum diluted at 1:100 and the titre of the ANA correlated with the highest serum dilution factor that allowed for observing the fluorescence. The nuclear patterns were observed using the Olympus BX51 fluorescence microscope and all the antibodies kits were purchased from Euroimmune Company (Germany).

\section{IVF/ICSI protocol}

A long pituitary down-regulation protocol was used in all the patients. A long-acting gonadotropin-releasing hormone agonist (GnRH-a, Diphereline; Ipsen Pharma Biotech, Signes, France) was injected intramuscularly in the mid-luteal phase of the preceding cycle of gonadotropin (Gn) stimulation. After the complete pituitary down-regulation, recombinant human FSH (Gonal-F, Merck Serono SA, Geneva,Switzerland) was injected for $\mathrm{COH}$ and urinary gonadotrophin (Menotrophins for Injection, LIVZON, China) was added in the late-follicle phase. Human chorionic gonadotropin (HCG, LIVZON, China) at a dose of 10000 IU was injected when at least two follicles had reached $18 \mathrm{~mm}$ in mean diameter or more than three follicles had reached $17 \mathrm{~mm}$, and the serum estradiol (E2) levels indicated more than $250 \mathrm{pg} /$ $\mathrm{ml} /$ mature follicle. The oocytes pick-up (OPU) was performed transvaginally $36 \mathrm{~h}$ after the HCG injection.

The selection of fertilisation program, IVF or ICSI, was based on the semen condition on the day of oocyte retrieval. The oocyte was considered to be fertilised normally if a second polar body was extruded or if two pronuclei (2PN) were observed 16 hours after insemination.

Embryo quality is primarily assessed by the day $3 \mathrm{em}-$ bryo grading system in our centre. The day 3 embryos were evaluated based on the number and size of their blastomeres and the degree of fragmentation as follows: Grade 1: 6-8 even, equally sized blastomeres without fragmentation of the blastomeres; Grade 2: 6-8 even, equally sized blastomeres, and less than $20 \%$ fragmentation of the blastomeres; Grade 3: 4-6 uneven or irregularly shaped blastomeres, and 20-50\% fragmentation of the blastomeres; Grade 4: the embryos are considered non-viable with more than $50 \%$ fragmentation or with even lysed, contracted or dark blastomeres. The embryos graded 1 and 2 were considered good-quality or perfect embryos, and the embryos graded 1, 2 and 3 were considered available embryos. Two to three good-quality embryos were initially selected for transfer (ET).

Progesterone supplementation by daily intramuscular injection of $40 \mathrm{mg} /$ day for the initial three days and $60 \mathrm{mg} /$ day for the following days (approximately three months) was administered from the day of the oocyte retrieval. Pregnancy was diagnosed by a positive blood test for $\beta$-hCG at 14 days after the embryo transfer. Clinical pregnancy was confirmed by the detection of a gestational sac with a foetal heartbeat by transvaginal ultrasound examination 14 days later.

\section{Statistical analysis}

The statistical analysis was performed using SPSS version 13 statistical software. The continuous data were described as the mean \pm sd and compared using the Mann-Whitney U rank sum test for two independent samples or the Wilcoxon signed ranks test for the paired samples. The rates were compared using the Chi square test and Fisher's Exact Test when appropriate. The differences in the P-value $<0.05$ were considered to be statistically significant.

\section{Results}

\section{Basal clinical characteristics}

The ANA + group and the ANA- group had no significant differences in the age, duration of infertility, BMI, basal hormone levels, days of ovarian stimulation, total Gn dose, serum E2 level and endometrial thickness on the day of the HCG injection (Table 1).

The Titre $\leq 1: 320$ subgroup and the Titre $>1: 320$ subgroup also showed no significant differences in the age, duration of infertility, BMI, basal hormone levels, days of ovarian stimulation, total Gn dose, serum E2 level and endometrial thickness on the day of the HCG injection (Table 2).

The ANA + cycles without P + A and the ANA + cycles with $\mathrm{P}+\mathrm{A}$ had similar days of ovarian stimulation, total Gn dose, serum E2 level and endometrial thickness on the day of the HCG injection (Table 3).

\section{Fertilisation and embryo development in the ANA + group and the ANA- group}

The proportion of cycles using the ICSI fertilisation program was similar in the ANA + and ANA- groups $(57.6 \%$ vs. $54.5 \%, \mathrm{p}=\mathrm{ns}$ ). The semen parameters (i.e., the semen volume, sperm concentration and progressive motility) on the day of OPU were not significantly different in the ICSI cycles and IVF cycles, respectively, between the two groups.

The MII oocytes rate $(78.1 \%$ vs. $82.6 \%)$ and the normal fertilisation rate in the ICSI/IVF cycles $(70.5 \%$ vs. $83.1 \%$, and $66.1 \%$ vs. $76.0 \%$ ) in the ANA + group were significantly lower than those in the ANA-group, whereas the opposite case occurred for the abnormal fertilisation rate 
Table 1 Basal characteristics in the ANA + group and the ANA- group

\begin{tabular}{lccc}
\hline Variables & ANA + group & ANA- group & P \\
\hline Patients & 66 & 233 & $\mathrm{~ns}$ \\
Age $(\mathrm{yrs})$ & $32.33+/-4.25$ & $31.41+/-3.84$ & $\mathrm{~ns}$ \\
BMI $\left(\mathrm{kg} / \mathrm{m}^{2}\right)$ & $21.72+/-2.58$ & $21.77+/-3.04$ & $\mathrm{~ns}$ \\
Duration of infertility (yrs) & $5.06+/-2.66$ & $4.73+/-3.10$ & $\mathrm{~ns}$ \\
bFSH (IU/L) & $7.15+/-1.84$ & $7.06+/-2.00$ & $\mathrm{~ns}$ \\
bLH (IU/L) & $4.61+/-2.85$ & $4.41+/-2.16$ & $\mathrm{~ns}$ \\
bE2 $($ pg/ml) & $46.41+/-19.60$ & $48.82+/-19.39$ & $\mathrm{~ns}$ \\
Days of ovarian stimulation & $12.21+/-2.36$ & $12.65+/-2.18$ & $\mathrm{~ns}$ \\
Total Gn dose (U) & $2464.77+/-78.71$ & $2399.25+/-803.25$ & $\mathrm{~ns}$ \\
E2 levels on HCG day (pg/ml) & $3014.28+/-1337.26$ & $2952.86+/-1401.69$ & $\mathrm{~ns}$ \\
Endometrial thickness on HCG day $(\mathrm{mm})$ & $10.46+/-1.64$ & $10.96+/-2.04$ & $\mathrm{~ns}$
\end{tabular}

$\mathrm{P}<0.05$ was considered to be statistically significant.

in the ICSI/IVF cycles $(4.91 \%$ vs. $0.36 \%$, and $4.33 \%$ vs. $2.33 \%)$. There were no significant differences between the groups in the cleavage rate and perfect and available embryo rates (Table 4).

\section{IVF/ICSI outcomes in the ANA + group and the}

\section{ANA- group}

The implantation rate (9.09\% vs. $36.8 \%)$ and the clinical pregnancy rate $(17.3 \%$ vs. $56.5 \%)$ in the ANA + group were significantly lower than those in the ANA- group, whereas the early miscarriage rate $(44.4 \%$ vs. $9.62 \%)$ showed the opposite result (Figure 1-a).

Fertilisation and embryo development in the titre $\leq 1: 320$ subgroup and the titre $>1: 320$ subgroup

The MII oocytes rate ( $73.6 \%$ vs. $80.4 \%)$ and the normal fertilisation rate in the ICSI/IVF cycle $(64.1 \%$ vs. $73.6 \%$ and $57.6 \%$ vs. $68.3 \%$ ) in the Titre $>1: 320$ subgroup were lower than those in the Titre $\leq 1: 320$ subgroup but without significance. The abnormal fertilisation rate in the
IVF cycles $(10.20 \%$ vs. $2.75 \%)$ in the Titre $>1: 320$ subgroup was higher than that in the Titre $\leq 1: 320$ subgroup. There were no significant differences between the subgroups in the cleavage rate and the perfect and available embryo rates (Table 5 ).

\section{IVF/ICSI outcomes in the titre $\leq 1: 320$ subgroup and the}

Titre $>$ 1:320 subgroup

No significant differences were observed between these subgroups in the implantation rate, clinical pregnancy rate and early miscarriage rate (Figure 1-b).

Fertilisation, embryo development and outcomes in the ANA + cycles without P + A and the ANA + cycles with $\mathrm{P}+\mathrm{A}$

The semen parameters (i.e., the semen volume, sperm concentration and progressive motility) on the day of OPU were not different significantly in the ICSI cycles and the IVF cycles, respectively, between the ANA + cycles without $\mathrm{P}+\mathrm{A}$ and the ANA + cycles with $\mathrm{P}+\mathrm{A}$.

Table 2 Basal characteristics in the titre $\leq 1: 320$ subgroup and the titre $>1: 320$ subgroup

\begin{tabular}{|c|c|c|c|}
\hline Variables & Titre $\leq 1: 320$ & Titre $>1: 320$ & $\mathbf{P}$ \\
\hline Patients & 46 & 20 & - \\
\hline Age (yrs) & $31.72+/-4.12$ & $33.75+/-4.30$ & ns \\
\hline$B M I\left(\mathrm{~kg} / \mathrm{m}^{2}\right)$ & $21.96+/-2.55$ & $21.16+/-2.63$ & ns \\
\hline Duration of infertility (yrs) & $4.98+/-2.65$ & $5.20+/-2.75$ & ns \\
\hline bFSH (IU/L) & $7.00+/-2.02$ & $7.49+/-1.33$ & ns \\
\hline$b L H(I U / L)$ & $4.68+/-3.11$ & $4.46+/-2.22$ & ns \\
\hline$b E 2(p g / m l)$ & $45.97+/-21.09$ & $47.43+/-16.12$ & ns \\
\hline Days of ovarian stimulation & $12.52+/-2.30$ & $11.50+/-2.40$ & ns \\
\hline Total Gn dose (U) & $2553.0+/-838.50$ & $2261.25+/-619.50$ & ns \\
\hline E2 levels on the HCG day $(\mathrm{pg} / \mathrm{ml})$ & $3017.61+/-1404.05$ & $3006.64+/-1203.62$ & ns \\
\hline Endometrial thickness on HCG day $(\mathrm{mm})$ & $10.40+/-1.74$ & $10.60+/-1.42$ & ns \\
\hline
\end{tabular}

$\mathrm{P}<0.05$ was considered to be statistically significant. 
Table 3 Basal characteristics in the ANA + cycles without P + A and the ANA + cycles with P + A

\begin{tabular}{|c|c|c|c|}
\hline Variables & Without $P+A$ & With $P+A$ & $\mathbf{P}$ \\
\hline Patients & 21 & 21 & - \\
\hline Age (yrs) & $31.71+/-3.61$ & - & - \\
\hline$B M I\left(\mathrm{~kg} / \mathrm{m}^{2}\right)$ & $21.53+/-2.23$ & - & - \\
\hline Duration of infertility (yrs) & $4.62+/-2.44$ & - & - \\
\hline bFSH (IU/L) & $7.38+/-2.10$ & - & - \\
\hline$b L H(I U / L)$ & $4.57+/-2.46$ & - & - \\
\hline$b E 2(p g / m l)$ & $52.85+/-24.40$ & - & - \\
\hline Days of ovarian stimulation & $12.22+/-2.14$ & $11.95+/-2.13$ & ns \\
\hline Total Gn dose $(U)$ & $2719.64+/-808.35$ & $2567.86+/-722.85$ & ns \\
\hline E2 levels on HCG day $(\mathrm{pg} / \mathrm{ml})$ & $2704.12+/-1305.23$ & $3139.39+/-1417.05$ & ns \\
\hline Endometrial thickness on HCG day $(\mathrm{mm})$ & $10.58+/-1.93$ & $10.59+/-2.59$ & ns \\
\hline
\end{tabular}

$\mathrm{P}<0.05$ was considered to be statistically significant.

After the prednisone plus low-dose aspirin adjuvant therapy, the number of $2 \mathrm{PN}(4.86+/-2.89$ vs. $7.05+/-3.17)$, embryos $(4.81+/-2.93$ vs. $6.90+/-3.24)$, perfect embryos $(3.24+/-2.41$ vs. $4.76+/-2.93)$ and available embryos $(3.62+/-2.22$ vs. $5.76+/-3.25)$, as well as the pregnancy rate $(12.5 \%$ vs. $57.1 \%)$ and the implantation rate $(6.06 \%$ vs. $27.9 \%)$ increased significantly. The early miscarriage rate was not statistically analysed because of the small number of cases (Table 6).

The mean number of embryos for transfer per cycle $(2.76+/-0.58$ vs. $2.06+/-0.44, \mathrm{P}=0.008)$ in the cycles with $\mathrm{P}+\mathrm{A}$ was higher than that in the cycles without

Table 4 Fertilisation and embryo development in the ANA + group and the ANA- group

\begin{tabular}{|c|c|c|c|}
\hline Variables & ANA + group & ANA- group & $\mathbf{P}$ \\
\hline OPU cycles (IVF + ICSI) & $66(28+38)$ & $233(106+127)$ & - \\
\hline ET cycles & 52 & 184 & ns \\
\hline Proportion of ICSI program (\%) & $57.6(38 / 66)$ & $54.5(127 / 233)$ & ns \\
\hline Semen volume (ml, ICSI cycles) & $3.46+/-0.74$ & $3.96+/-0.86$ & ns \\
\hline Sperm concentration ( $\times 106 / \mathrm{ml}$, ICSI cycles) & $16.86+/-13.34$ & $15.45+/-11.23$ & ns \\
\hline Sperm progressive motility (\%, ICSI cycles) & $11.97+/-9.74$ & $10.96+/-8.44$ & ns \\
\hline Semen volume (ml, IVF cycles) & $3.67+/-0.87$ & $3.86+/-0.84$ & ns \\
\hline Sperm concentration ( $\times 106 / m l$, IVF cycles) & $65.33+/-44.33$ & $68.84+/-46.00$ & ns \\
\hline Sperm progressive motility (\%, IVF cycles) & $40.28+/-16.78$ & $35.23+/-15.84$ & ns \\
\hline Retrieved oocytes & $9.73+/-5.03$ & $10.38+/-4.15$ & ns \\
\hline MII oocytes rate (\%, ICSI cycle) & $78.1(285 / 365)$ & $82.6(1112 / 1346)$ & 0.047 \\
\hline Normal fertilisation rate (\%, ICSI cycle) & $70.5(201 / 285)$ & $83.1(924 / 1112)$ & $<0.001$ \\
\hline Abnormal fertilisation rate (\%, ICSI cycle) & $4.91(14 / 285)$ & $0.36(4 / 1112)$ & $<0.001$ \\
\hline Normal fertilisation rate (\%, IVF cycle) & $66.1(183 / 277)$ & $76.0(815 / 1073)$ & $<0.001$ \\
\hline Abnormal fertilisation rate (\%, IVF cycle) & $4.33(12 / 277)$ & $2.33(23 / 1073)$ & 0.041 \\
\hline Cleavage rate (\%) & $97.7(375 / 384)$ & $98.5(1713 / 1739)$ & ns \\
\hline Perfect embryo rate (\%) & $63.7(239 / 375)$ & $66.5(1139 / 1713)$ & ns \\
\hline Available embryo rate (\%) & $78.7(295 / 375)$ & $82.9(1420 / 1713)$ & ns \\
\hline Transferred embryos/cycle & $2.12 \pm 0.55$ & $2.11 \pm 0.38$ & ns \\
\hline
\end{tabular}

$\mathrm{P}<0.05$ was considered to be statistically significant.

Note: Sperm with progressive motility, including those that move fast in a straight line (grade a) and that move forward but tend to travel in a curved or bent motion (grade b). MII oocytes rate $=\mathrm{M} \mathrm{II} \mathrm{oocytes/total} \mathrm{oocytes} \mathrm{in} \mathrm{the} \mathrm{ICSI} \mathrm{cycle;} \mathrm{normal} \mathrm{fertilisation} \mathrm{rate}=2 \mathrm{PNs} /$ total M II oocytes in the ICSI cycle or 2PNs/total oocytes in the IVF cycle; abnormal fertilisation rate $=1 \mathrm{PNs}+$ multi-PNs/total M II oocytes in the ICSI cycle or 1PNs + multi-PNs/total oocytes in the IVF cycle. 

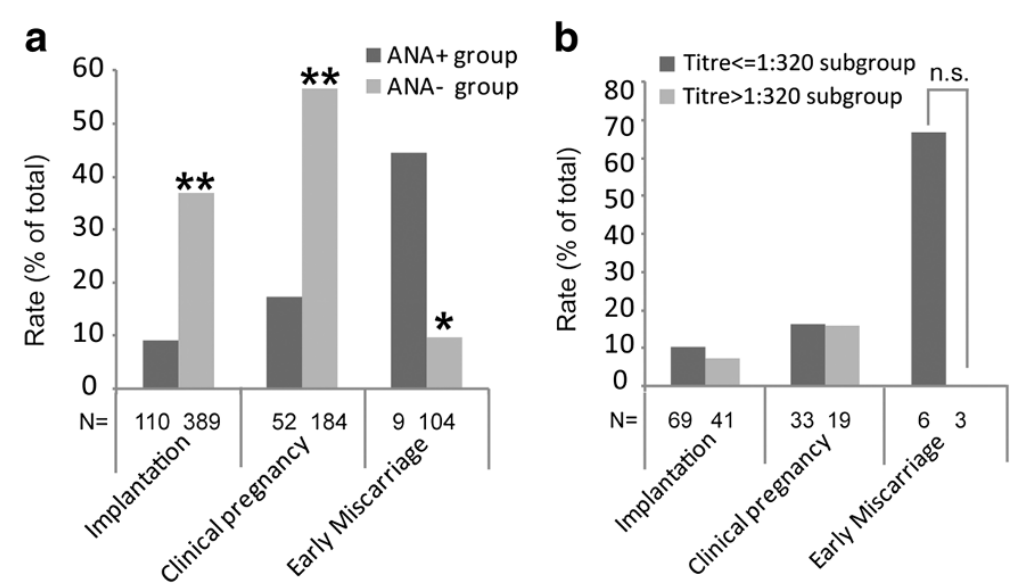

Figure 1 Pregnancy outcome in IVF/ICSI cycles. (a) The IVF/ICSI outcome in the ANA + group and the ANA-group. In the ANA + group, the implantation rate $(9.09 \%$ vs. $36.8 \%)$ and the clinical pregnancy rate (17.3\% vs. $56.5 \%)$ decreased significantly, and the early miscarriage rate (44.4\% vs. 9.62\%) increased markedly more than in the ANA- group. (b) The IVF/ICSI outcome in the Titre $\leq 1: 320$ subgroup and the Titre > 1:320 subgroup. No significant differences were found between the two subgroups in the implantation rate, the clinical pregnancy rate and the early miscarriage rate. ${ }^{*} \mathrm{P}<0.05,{ }^{* *} \mathrm{P}<0.01$.

$\mathrm{P}+\mathrm{A}$ (Table 6). The increased pregnancy rate in the cycles with $\mathrm{P}+\mathrm{A}$ might be because of the medication effect and/or the greater number of embryos for transfer per cycle. It appeared that the pregnancy rate was not an appropriate indicator for evaluating the medication effect.

\section{Discussion}

For several years, detection of autoantibodies has been recommended in clinical practice for women with infertility including the detection of ANA, APA and ATA. A link of APA with recurrent pregnancy loss has been established, and treatment based on anticoagulation such as subcutaneous heparin is effective [3]. As an organ-specific autoantibody, ATA affect pregnancy outcome negatively by damaging the thyroid function, and the thyroxin replacement therapy is efficacious in preventing foetal loss for patients with recurrent miscarriage $[1,2]$. However, the potential correlation between ANA and IVF/ICSI outcome and specific medication are less reported.

Previous reports showed that ANA were relevant to adverse reproductive events including recurrent spontaneous abortion, endometriosis, infertility, IVF failure and ovarian dysfunction [6-10]. One recent study proposed that ANA might impair oocyte quality and embryo development, leading to reduced pregnancy and implantation rates [10]. A further exploration [20] similarly suggested that IVF outcomes were markedly poorer in ANA + women, and this effect became worse with an increased level of serum ANA.

In this study, we found that the presence of ANA in serum predicted an adverse IVF/ICSI outcome, primarily

Table 5 Fertilisation and embryo development in the titre $\leq 1: 320$ subgroup and the titre $>1: 320$ subgroup

\begin{tabular}{|c|c|c|c|}
\hline Variables & Titre $\leq 1: 320$ & Titre $>1: 320$ & $\mathbf{P}$ \\
\hline OPU cycles & 46 & 20 & - \\
\hline ET cycles & 33 & 19 & - \\
\hline Retrieved oocytes & $9.96+/-5.04$ & $9.20+/-5.08$ & ns \\
\hline MII oocytes rate (\%, ICSI cycle) & $80.4(193 / 240)$ & $73.6(92 / 125)$ & ns \\
\hline Normal fertilisation rate (\%, ICSI cycle) & $73.6(142 / 193)$ & $64.1(59 / 92)$ & ns \\
\hline Abnormal fertilisation rate (\%, ICSI cycle) & $5.18(10 / 193)$ & $4.35(4 / 92)$ & ns \\
\hline Normal fertilisation rate (\%, IVF cycle) & $68.3(149 / 218)$ & $57.6(34 / 59)$ & ns \\
\hline Abnormal fertilisation rate (\%, IVF cycle) & $2.75(6 / 218)$ & $10.2(6 / 59)$ & 0.034 \\
\hline Cleavage rate (\%) & $97.6(284 / 291)$ & $97.8(91 / 93)$ & ns \\
\hline Perfect embryo rate (\%) & $63.7(181 / 284)$ & $63.7(58 / 91)$ & ns \\
\hline Available embryo rate (\%) & $78.2(222 / 284)$ & $80.2(73 / 91)$ & ns \\
\hline Transferred embryos/cycle & $2.09+/-0.46$ & $2.16+/-0.69$ & ns \\
\hline
\end{tabular}

$\mathrm{P}<0.05$ was considered to be statistically significant. 


\begin{tabular}{|c|c|c|c|}
\hline Variables & Without $P+A$ & With $P+A$ & $\mathbf{P}$ \\
\hline OPU cycles (IVF + ICSI) & $21(11+10)$ & $21(11+10)$ & - \\
\hline ET cycles & 16 & 21 & - \\
\hline Semen volume (mI, ICSI cycles) & $3.54+/-0.81$ & $3.09+/-0.87$ & ns \\
\hline Sperm concentration ( $\times 106 / \mathrm{ml}$, ICSI cycles) & $14.64+/-11.21$ & $16.34+/-10.65$ & ns \\
\hline Sperm progressive motility (\%, ICSI cycles) & $12.87+/-10.97$ & $15.34+/-11.56$ & ns \\
\hline Semen volume (ml, IVF cycles) & $3.44+/-0.93$ & $3.64+/-0.83$ & ns \\
\hline Sperm concentration (×106/ml, IVF cycles) & $62.56+/-43.78$ & $66.23+/-39.23$ & ns \\
\hline Sperm progressive motility (\%, IVF cycles) & $46.89+/-15.45$ & $43.89+/-17.68$ & ns \\
\hline Retrieved oocytes & $8.10+/-3.95$ & $9.24+/-4.10$ & ns \\
\hline MIl oocytes & $6.50+/-4.09$ & $7.20+/-3.99$ & ns \\
\hline Two pro-nuclei & $4.86+/-2.89$ & $7.05+/-3.17$ & 0.004 \\
\hline Perfect embryos & $3.24+/-2.41$ & $4.76+/-2.93$ & 0.037 \\
\hline Available embryos & $3.62+/-2.22$ & $5.76+/-3.25$ & 0.008 \\
\hline Embryos & $4.81+/-2.93$ & $6.90+/-3.24$ & 0.006 \\
\hline Transferred embryos/cycle & $2.06+/-0.44$ & $2.76+/-0.58$ & 0.008 \\
\hline Pregnancy rate (\%) & $12.5(2 / 16)$ & $57.1 \%(12 / 21)$ & 0.006 \\
\hline Implantation rate (\%) & $6.06(2 / 33)$ & $27.9(16 / 58)$ & 0.013 \\
\hline Early miscarriage rate (\%) & $100(2 / 2)$ & $25(3 / 12)$ & - \\
\hline
\end{tabular}

$\mathrm{P}<0.05$ was considered to be statistically significant.

reflecting the lower rates of MII oocytes and normal fertilisation and the reduced rates of implantation and clinical pregnancy, as well as the increased rates of abnormal fertilisation and early miscarriage. The cleavage rate and the perfect and available embryo rates were not significantly different between the ANA + women and the controls, which was a finding that did not coincide with that of Ying $\mathrm{Y}$ et al. [10]. We found that ANA-titre $\leq 1: 320$ women had as poor IVF/ICSI outcomes as the ANA-titre $>1: 320$ women, and only the abnormal fertilisation rate in the IVF cycles in the ANA-titre $>1: 320$ women was significantly higher. These results did not suggest the titre-dependent effect of ANA on IVF/ICSI outcomes, in agreement with Hasegawa et al. [21] and Taniguchi et al. [22].

In general terms, abnormal autoimmune conditions may impair all stages of fertility, leading to ovarian and testicular failure, implantation failure or pregnancy loss through different putative mechanisms [5]. It has been proposed that APA and A- $\beta 2-$ GPI could result in thrombosis of placental blood vessels, dysfunctions of trophoblasts in the peri-implantation period or an imbalance of maternal hormones [23-25]. Unlike APA and A- $\beta 2-G P I$, the mechanism by which ANA determine reproductive failure remains speculative. Hasegawa et al. [21] held that ANA did not target specific organs and only presented as an abnormal degree of autoimmunity, based on their findings that the adverse IVF outcome was not ANA-titre dependent. One recent well designed study [26] demonstrated that the presence of APA, ANA or ATA in recipients using donor oocytes had no negative effect on pregnancy, implantation or miscarriage, which suggested that implantation failure might be because of poor-quality oocytes that may lead to a subsequent embryo development disorder. One in vivo test [27], in which anti-centromere antibodies were microinjected into mouse oocytes, showed the anticentromere antibody could interfere with chromosome congression in the pro-metaphase. Shirota $\mathrm{K}$ et al. [18] considered that anti-centromere antibodies might infiltrate oocytes and lead to centromere dysfunction during meiosis and mitosis and impair the transition from MI to MII during oocyte maturation. Ying Y et al. [20] has confirmed that ANA exist in follicular fluid and embryos in ANA + patients, and serum and follicular fluid ANA negatively correlated with the number of high-quality embryos. The embryos co-cultured with IgG extracted from ANA + women were found to be severely impaired or even died [19].

Ando et al. [28] administered low-dose prednisolone $(5 \mathrm{mg} / \mathrm{d})$ or dexamethasone $(0.5 \mathrm{mg} / \mathrm{d})$ daily during the entire IVF cycle until the pregnancy test was performed in 51 lVF-ET cycles of patients positive for ANA, antiDNA antibody, and/or lupus anti-coagulant (LAC), as well as 29 IVF-ET cycles of patients negative for any antibodies and discovered significant increases of 
pregnancy and implantation rates in the antibodiespositive patients with corticosteroid treatment but not in the antibody-negative patients. Hasegawa et al. [21] administered prednisolone $(10 \mathrm{mg} / \mathrm{d})$ plus low-dose aspirin $(81 \mathrm{mg} / \mathrm{d})$ to ANA + and/or APA + women from the first day of $\mathrm{COH}$ until pregnancy was confirmed by ultrasonography and discovered that the ANA + women with treatment had significantly better outcomes of IVFET (40.6\% pregnancy rate and $20.3 \%$ implantation rate). Taniguchi et al. [22] administered prednisolone (15-60 $\mathrm{mg} / \mathrm{d}$ ) starting from the first day after OPU for 5 days in 56 IVF-ET cycles of 24 ANA + women and 167 IVF-ET cycles of 96 ANA-women and found the implantation rate and clinical pregnancy rate improved significantly in the ANA + woman but not in the ANA-women, which coincides with the findings of Ando et al. One previous prospective study administered prednisone plus aspirin for 4 weeks before IVF treatment to 52 women positive for ACA, ANA, anti-dsDNA antibody, rheumatoid factor, and/or LAC and ultimately obtained a satisfactory clinical pregnancy rate (32.7\%) [29]. The present study pretreated ANA + women with prednisone $(10 \mathrm{mg} / \mathrm{d})$ plus low-dose aspirin $(100 \mathrm{mg} / \mathrm{d})$ (i.e., $\mathrm{P}+\mathrm{A})$ for three months before IVF treatment and observed that the ANA + cycles with $\mathrm{P}+\mathrm{A}$ had markedly more $2 \mathrm{PN}$, high-quality and available embryos, and an increased implantation rate. Our study also showed that the ANA titre was not relevant to the IVF/ICSI outcome and the adjuvant treatment was not closely related to the reduction of the serum ANA titre, which is in agreement with Hasegawa et al. [21]. In this study and that of Hasegawa et al. [21], women positive for autoantibodies were administered a 10-mg daily dose of prednisone or prednisolone, and this dosage is too low to reduce the autoantibody titres. Thus the corticosteroid effect may be derived from another mechanism such as an antiinflammatory action or the regulation of immune cells as in the reduction of NK cells [21]. Low-dose aspirin for its anti-thrombotic effect may reduce uterine and intraovarian vascular resistance, improve blood perfusion and increase oocyte maturation, the high-quality embryos rate, and the implantation rate [21]. Although these five studies including the present work demonstrated a beneficial effect of corticosteroid or corticosteroid plus aspirin therapy, an ideal protocol for this adjuvant therapy (i.e., the indication of the patients, the drug selection and dosage, and the timing of commencement and end) requires further investigation.

In this study, we observed the detrimental effect of ANA on IVF/ICSI outcome and the beneficial effect of prednisone plus low-dose aspirin adjuvant treatment for ANA + patients. The comparison between the first IVF/ ICSI cycle to a subsequent one would unavoidably yield bias, which is the limitation of our study. To minimise the bias, we rigorously selected the subjects, and there was a long interval between the first and the second IVF/ICSI cycles of at least 3 months. In our future work, we will attempt to perform a placebo-controlled, double blind, and prospective study.

\section{Conclusions}

These observations suggest that ANA could exert a detrimental effect on the IVF/ICSI outcome that may not be titre-dependent, and prednisone plus low-dose aspirin adjuvant treatment could be useful in ANA + patients. This hypothesis should be verified in placebo-controlled, double blind, and prospective studies.

\section{Abbreviations}

A: Aspirin; ACA: Anti-cardiolipin antibody; A-B2-GPI: Anti-beta(2)-glycoprotein l; ANA: Anti-nuclear antibody; APA: Anti-phospholipids antibody; ATA: Antithyroid antibody; anti-dsDNA antibody: Anti-double strand DNA antibody; $\mathrm{COH}$ : Controlled ovarian stimulation; ET: Embryo transfer; E2: Estradiol; Gn: Gonadotropin; GnRH: Gonadotropin-releasing hormone; HCG: Human chorionic gonadotropin; ICSI: Intracytoplasmic sperm injection; IFT: Indirect immunofluorescence method; IVF: In vitro fertilisation; LAC: Lupus anticoagulant; OPU: Oocytes pick-up; P: Prednisone; STD: Sexually transmitted diseases; T3: Triiodothyrorine; T4: Thyroxine; TSH: Thyroid stimulating hormone; 2PN: Two pro-nuclei.

\section{Competing interests}

The authors declare that they have no competing interests.

\section{Authors' contributions}

QZ and LW conducted the data collection, participated in the design of the study, and drafted the manuscript. BX and $\mathrm{MHH}$ participated in the processing of the data and the statistical analysis, and performed the diagramming. XHT and $\mathrm{JJ}$ J conducted the interpretation of the data and the revision for important intellectual content and grammar. YSL conceived of the study, participated in its design and coordination, and helped to draft the manuscript. All the authors read and approved the final manuscript.

\section{Acknowledgements}

The authors would like to thank Dr. Lars Johansson of the Centre for Assisted Reproduction, Codra Hospital, Podgorica, Montenegro, for revising the English in the manuscript. Additionally, a professional service (http://webshop.elsevier. com/languageediting) was used for language corrections.

Received: 21 June 2013 Accepted: 3 October 2013

Published: 5 October 2013

\section{References}

1. Vaquero E, Lazzarin N, Caserta D, Valensise H, Baldi M, Moscarini M, Arduini $D$ : Diagnostic evaluation of women experiencing repeated in vitro fertilization failure. Eur J Obstet Gynecol Reprod Biol 2006, 125:79-84.

2. Revelli A, Casano S, Piane LD, Grassi G, Gennarelli G, Guidetti D, Massobrio $\mathrm{M}$ : A retrospective study on IVF outcome in euthyroid patients with anti-thyroid antibodies: effects of levothyroxine, acetyl-salicylic acid and prednisolone adjuvant treatments. Reprod Biol Endocrinol 2009, 27:131-137.

3. Ruiz-Irastorza G, Crowther M, Branch W, Khamashta M: Antiphospholipid syndrome. Lancet 2010, 376:1498-1509.

4. Zhong YP, Ying Y, Wu HT, Zhou CQ, Xu YW, Wang Q, Li J, Shen XT, Li J: Relationship between Antithyroid antibody and pregnancy outcome following in vitro fertilization and embryo transfer. Int J Med Sci 2012, 9:121-125.

5. Carp HJ, Selmi C, Shoenfeld Y: The autoimmune bases of infertility and pregnancy loss. J Autoimmun 2012, 38:266-274.

6. Kaider AS, Kliider BD, Junowicz PB, Rousseu RG: Immunodiagnostic evaluation in women with reproductive failure. Am J Reprod Immunol 1999, 42:335-346. 
7. Kikuchi K, Shibahara H, Hirano Y, Kohno T, Hirashima C, Suzuki T, Takamizawa S, Suzuki M: Antinuclear antibody reduces the pregnancy rate in the first IVF-ET treatment cycle but not the cumulative pregnancy rate without specific medication. Am J Reprod Immunol 2003, 50:363-367.

8. Dias JA Jr, de Oliveira RM, Abrao MS: Antinuclear antibodies and endometriosis. Int J Gynaecol Obstet 2006, 93:262-263.

9. Ticconi C, Rotondi F, Veglia M, Pietropolli A, Bernardini S, Ria F, Caruso A, Di Simone N: Antinuclear autoantibodies in women with recurrent pregnancy loss. Am J Reprod Immunol 2010, 64:384-392.

10. Ying Y, Zhong YP, Zhou CQ, Xu YW, Wang Q, Li J, Shen XT, Wu HT: Antinuclear antibodies predicts a poor IVF-ET outcome: impaired Egg and embryo development and reduced pregnancy rate. Immunol Invest 2012, 41:458-468.

11. Fragkou IA, Mavrogianni VS, Cripps PJ, Gougoulis DA, Fthenakis GC: The bacterial florain the teat duct of ewes can protect against and can cause mastitis. Vet Res 2007, 38:525-545.

12. Ramírez-Sandoval R, Sánchez-Rodríguez SH, Herrera-van Oostdam D, Avalos-Díaz E, Herrera-Esparza R: Antinuclear antibodies recognize cellular autoantigens driven by apoptosis. Joint Bone Spine 2003, 70:187-194.

13. Cline AM, Radic MZ: Murine lupus autoantibodies identify distinct subsets of apoptotic bodies. Autoimmunity 2004, 37:85-93.

14. Gleicher N, el Roeity A, Confino E, Friberg J: Reproductive failure because of autoantibodies: unexplained infertility and pregnancy wastage. Am J Obstet Gynecol 1989, 160:1307-1385.

15. Reichlin M: Cellular dysfunction induced by penetration of autoantibodies into living cells: cellular damage and dysfunction mediated by antibodies to dsDNA and ribosomal P proteins. J Autoimmun 1998, 11:557-561.

16. Sun KH, Tang SJ, Lin ML, Wang YS, Sun GH, Liu WT: Monoclonal antibodies against human ribosomal $P$ proteins penetrate into living cells and cause apoptosis of Jurkat T cells in culture. Rheumatology 2001, 40:750-756.

17. Putterman C: New approaches to the renal pathogenicity of anti-DNA antibodies in systemic lupus erythematosus. Autoimmun Rev 2004, 3:7-11.

18. Shirota K, Nagata Y, Honjou K, Tsujioka H, Yoshizato T, Miyamoto S: Involvement of anticentromere antibody in interference with oocyte meiosis and embryo cleavage. Fertil Steril 2011, 95:2729-2731.

19. Kaider BD, Coulam CB, Roussev RG: Murine embryos as a direct target for some human autoantibodies in vitro. Hum Reprod 1999, 14:2556-2561.

20. Ying $Y$, Zhong YP, Zhou CQ, Xu YW, Ding CH, Wang Q, Li J, Shen XT: A further exploration of the impact of antinuclear antibodies on in vitro fertilization-embryo transfer outcome. Am J Reprod Immunol 2013, 70:221-229.

21. Hasegawa I, Yamanoto Y, Suzuki M, Murakawa H, Kurabayashi T, Takakuwa K, Tanaka K: Prednisolone plus low-dose aspirin improves the implantation rate in women with autoimmune conditions who are undergoing in vitro fertilization. Fertil Steril 1998, 70:1044-1048.

22. Taniguchi F: Results of Prednisolone given to improve the outcome of in vitro fertilization-embryo transfer in women with antinuclear antibodies. J Reprod Med 2005, 50:383-388.

23. Gharavi AE, Cucurull E, Tang H, Silver RM, Branch DW: Effect of antiphospholipid antibodies on beta (2) glycoprotein I-phospholipid interaction. Am J Reprod Immunol 1998, 39:310-315.

24. Chamley LW, Duncalf AM, Mitchell MD, Johnson PM: Action of anticardiolipin and antibodies to beta2-glycoprotein-I on trophoblast proliferation as a mechanism for fetal death. Lancet 1998, 352:1037-1038.

25. Katsuragawa H, Kanzaki H, Inoue T, Hirano T, Mori T, Rote NS: Monoclonal antibody against phosphatidylserine inhibits in vitro human trophoblastic hormone production and invasion. Biol Reprod 1997 56:50-58.

26. Chantilis SJ, Kutteh WH, Blankenship L, Potter CH, Ward D, Davidson T: Antiphospholipid (APA), antinuclear (ANA), and antithyroid (ATA) antibodies do not affect pregnancy outcome in oocyte donation recipients. Fertil Steril 2008, 90:S388.

27. Simerly C, Balczon R, Brinkley BR, Schatten G: Microinjected kinetochore antibodies interfere with chromosome movement in meiotic and mitotic mouse oocytes. J Cell Biol 1990, 111:1491-1504.
28. Ando T, Suganuma N, Furuhashi M, Asada Y, Kondo I, Tomoda Y: Successful glucocorticoid treatment for patients with abnormal autoimmunity on in vitro fertilization and embryo transfer. J Assist Reprod Genet 1996, 13:776-781.

29. Geva E, Amit A, Lerner-Geva L, Yaron Y, Daniel Y, Schwartz T, Azem F, Yovel I, Lessing JB: Prednisone and aspirin improve pregnancy rate in patients with reproductive failure and autoimmune antibodies: a prospective study. Am J Reprod Immunol 2000, 43:36-40.

doi:10.1186/1477-7827-11-98

Cite this article as: Zhu et al:: A retrospective study on IVF/ICSI outcome in patients with anti-nuclear antibodies: the effects of prednisone plus low-dose aspirin adjuvant treatment. Reproductive Biology and Endocrinology 2013 11:98.

\section{Submit your next manuscript to BioMed Central and take full advantage of:}

- Convenient online submission

- Thorough peer review

- No space constraints or color figure charges

- Immediate publication on acceptance

- Inclusion in PubMed, CAS, Scopus and Google Scholar

- Research which is freely available for redistribution 